INSTITUT NATIONAL DE LA STATISTIQUE ET DES ETUDES ECONOMIQUES

Série des Documents de Travail du CREST

(Centre de Recherche en Economie et Statistique)

\title{
$n^{\circ} 2006-06$
}

\section{Assessing Horizontal Mergers under Uncertain Efficiency Gains*}

\author{
Ph. CHONÉ \\ L. LINNEMER
}

Les documents de travail ne reflètent pas la position de l'INSEE et n'engagent que leurs auteurs.

Working papers do not reflect the position of INSEE but only the views of the authors.

\footnotetext{
* We are grateful to Christian Gollier, Hannu Vartiainen and Thibaud Vergé for their comments as well as to the participants of the Area Conference on Applied Microeconomics, Munich (CESifo), March 2006.

${ }^{1}$ CREST-LEI, 28 rue des Saints-Pères, 75007 Paris. Email : chone@ensae.fr

${ }^{2}$ CREST-LEI, 28 rue des Saints-Pères, 75007 Paris. Email : Laurent.linnemer@ensae.fr (contact author)
} 


\title{
Assessing horizontal mergers under uncertain efficiency gains*
}

\author{
Philippe Chonétand Laurent Linnemer ${ }^{\ddagger}$
}

April 2006

\footnotetext{
${ }^{*}$ We are grateful to Christian Gollier, Hannu Vartiainen and Thibaud Vergé for their comments as well as to the participants of the Area Conference on Applied Microeconomics, Munich (CESifo), March 2006.

${ }^{\dagger}$ CREST-LEI, 28 rue des Saints Pères, 75007 Paris. Email : chone@ensae.fr

${ }^{\ddagger}$ CREST-LEI, 28 rue des Saints Pères, 75007 Paris. Email : laurent.linnemer@ensae.fr (contact author)
} 


\begin{abstract}
The analysis of horizontal mergers hinges on a tradeoff between unilateral effects and efficiency gains. The article examines the role of uncertainty (on the efficiency gains) in this tradeoff. Common wisdom is that the antitrust authorities should be very cautious about random gains. Our results show that dismissing efficiency gains on the sole ground that they are uncertain would not be theoretically founded. Indeed, the attitude towards uncertainty depends on the curvature of the social objective function. We exhibit a number of situations where the objective is convex in the efficiency gains, implying that competition authorities should welcome the risk for a given expectation of efficiency gains. Implications for empirical merger analysis are exposed.
\end{abstract}

Keywords: Merger analysis, Antitrust, Efficiency gains, Uncertainty.

JEL Classification: K210, L120, L410

\title{
Résumé
}

L'analyse des fusions horizontales repose sur un arbitrage entre effets unilatéraux et gains d'efficacité. Cet article examine le rôle de l'incertitude (sur les gains d'efficacité) dans cet arbitrage. Les autorités de concurrence sont généralement très méfiantes vis-à-vis de gains aléatoires. Nos résultats montrent que rejeter des gains d'efficacité au seul motif qu'ils sont incertains ne serait pas théoriquement fondé. En effet, l'attitude vis-à-vis du risque dépend de la courbure de la fonction objectif. Nous mettons en évidence de nombreuses situations où l'objectif est convexe en les gains d'efficacité, et où, en conséquence, les autorités de concurrence devraient accueillir favorablement le risque (à espérance donnée). Des conséquences pour l'analyse empirique des fusions sont présentées.

Mots clefs : Fusion horizontale, politique de la concurrence, gains d'efficacité, incertitude.

JEL Classification : K210, L120, L410 


\section{Introduction}

This article reexamines the welfare tradeoffs put forward by Williamson (1968) when efficiency gains are uncertain. We focus on horizontal mergers that create market power, but at the same time yield cost economies (or losses) of random magnitude. In the modern antitrust langage, the variation of Nash equilibrium prices following the alteration of market structure is often referred to as "unilateral effects". These effects have been recognized for a long time by U.S competition authorities. Section 2.2 of the 1992 Horizontal Merger guidelines is entitled "Lessening of competition through unilateral effects". On the other side of the Atlantic ocean, the 1989 European Community Merger Regulation had been criticized by economists and practitioners because it was not clear that the prohibition criterion (creation or strengthening of a dominant position) encompassed unilateral effects. In January 2004, the substantive test has been reworded, so as to unambiguously fill the gap.

As regards efficiencies, it is fair to say that they are now widely taken into account by competition authorities, even though differences in the standards of proof may subsist. Section 4 of the U.S. Merger Guidelines, which is devoted on efficiencies and has been revised in 1997, recognizes that "the primary benefit of mergers to the economy is their potential to generate such efficiencies" and acknowledges their potentially pro-competitive impact: "Efficiencies generated through merger can enhance the merged firm's ability and incentive to compete, which may result in lower prices, improved quality, enhanced service, or new products." In Europe, a rather skeptical view about efficiency gains seems to prevail. The 2004 EC Regulation imposes high standards regarding efficiency gains which are referred to as "development of technical and economic progress": they are taken into account only "provided that it is to consumers' advantage and does not form an obstacle to competition." Having opened the scope for an efficiency defence, competition authorities emphasize the issue of uncertainty:

Efficiencies are difficult to verify and quantify, in part because much of the information relating to efficiencies is uniquely in the possession of the merging firms. Moreover, efficiencies projected reasonably and in good faith by the merging firms may not be realized.

\section{(U.S. Merger Guidelines sec. 4)}

This quotation highlights two distinct informational features: asymmetry and imperfection. First, merging parties, arguably, know more about potential efficiency gains than competition authorities. Second, even if all the information known to the merging firms were available to the authorities, there would remain a lot of uncertainty about the realization and the magnitude of the efficiency gains. During the merger implementation, firms try to achieve cost reductions but this process is random and more or less successful. Efficiency losses (caused, for instance, by clashes between corporate cultures) cannot even be ruled out. 
Departing from most of the existing literature, which has studied implementation issues under asymmetric information (see for instance Besanko and Spulber (1993)), we concentrate on imperfect information in a simple framework where (i) the decision to merge is exogenous, as well as the efficiency gains; (ii) the objectives of society and of the competition authority are perfectly aligned and (iii) the expectation of a weighted sum of consumers' surplus and firms' profits is maximized. Following Deneckere and Davidson (1985), most of the analysis considers mergers in a multi-product industry under price differentiation and constant marginal costs of production. To check the robustness of our results, we examine some cases with homogenous goods, in particular Cournot competition, as in Farrell and Shapiro (1990).

In general, the objective is a non linear function of the efficiency gains. Even though society is risk-neutral with respect to revenues and utility, it should not be indifferent to the uncertainty affecting efficiencies. To understand the social attitude towards this risk, the article endeavors to characterize the curvature of the objective.

Surprisingly, consumers' surplus and firms' profits turn out to be convex functions of efficiency gains in many situations, implying that uncertainty should be welcomed by competition authorities. In particular, this is true as soon as demand functions are linear in prices, for any merger and any pre- and post-merger market structure, with price competition as well as with Cournot competition. No (e.g. symmetry) assumption on top of the linearity is required for this result to hold. Turning to nonlinear demand functions, we specialize to two polar cases: mergers to monopoly (strong unilateral effects) and Bertrand competition with homogenous products (no unilateral effect). In the former case, the monopoly profit is always convex; symmetry and an additional restriction (met, for instance, by CES demand systems) are needed for consumers' surplus to be convex. For a Logit demand system, total welfare is convex in efficiency gains while consumers' surplus can be either concave or convex depending on the value of the underlying parameters. Under Bertrand competition (and any demand function), consumers' surplus cannot raise after a merger. Yet, uncertainty might still be welcomed if the antitrust authorities put enough weight on firms' profits. Finally, when firms compete in quantity and costs are asymmetric, the consumers' surplus is convex in efficiency gains for a large class of demand functions (and for an arbitrary number of outsiders).

These examples certainly do not imply that the convexity property is generic. The curvature of the social objective depends on the specifics of each case, in particular on the functional form of the demand system. Yet, our analysis shows that the specification chosen in econometric studies may condition the implicit attitude towards uncertain efficiency gains. We elaborate on the implications of our results for empirical merger analysis in the last section.

The remainder of the article is organized as follows. Section 2 presents the competition framework in which the merger takes place and discusses the merger assessment under uncertainty about efficiency gains. Section 3 solves the linear case. Section 4 analyzes mergers to monopoly under few restrictions on demands. Section 5 deals with Bertrand competition with homogeneous products. Section 6 extends the analysis to Cournot competition. 
Section 7 presents some implications of the results for merger control in practice, with a particular emphasis on the use of econometric models.

\section{Framework}

In this section, we introduce various notations and assumptions to describe a merger in a multi-products firms industry under constant marginal costs of production.

Competitive environment: Let $N=\{1, \ldots, n\}$ denote the set of all brands. We use the index $I$ for the merging parties and $J$ for the outsiders. The structure of the industry before the merger is described by a partition of $N$ into $l+r$ subsets: $\left\{I_{1}, \ldots, I_{l}, J_{1}, \ldots, J_{r}\right\}$.

The structure of the industry after the merger is described by a partition of $N$ in $1+r$ firms: $\left\{I, J_{1}, \ldots, J_{r}\right\}$, where $I=\bigcup_{i=1}^{l} I_{i}$ denotes the set of brands owned by the merged entity. Thus, $l$ is the number of merging parties, $r$ the number of outsiders.

Costs and efficiency gains: Before and after the merger, all goods are produced at constant marginal cost. Let $c_{k}(k=1$ to $n)$ denote the marginal cost of brand $k$ before the merger. Without any loss of generality, the post-merger marginal costs of the merging firms can be noted: $\left(1-\gamma_{i}\right) c_{i}$, with $\gamma_{i} \leq 1$.

In most of the paper, we suppose, for simplicity, that efficiency gains are one-dimensional, ${ }^{1}$ that is, they can be represented by a real-valued random variable $\gamma$. We do not want, however, to assume that the gains are proportional to the pre-merger marginal cost $c_{i}$ of each brand $i$. To avoid this restriction, we introduce nonnegative numbers $\lambda_{i}$ with $\max _{I} \lambda_{i}=1$, and assume that the gains are proportional to $\lambda_{i} c_{i}$. In other words, the post-merger marginal costs of the new entity are

$$
c_{i}(\gamma)=\left(1-\lambda_{i} \gamma\right) c_{i}, \quad i \in I
$$

If each variety benefits similarly from the efficiency gains, then $\lambda_{i}=1$ for all $i$. But marginal costs might vary differently: for instance, only one product could benefit from the efficiencies, that is, only one $\lambda_{i}$ could be positive.

Since the costs of each variant must remain nonnegative, we have: $\gamma \leq 1$. The merger allows to reduce marginal costs when $0<\gamma \leq 1$; it deteriorates the productivity when $\gamma<0$ (efficiency losses). The value of the random variable $\gamma$ is realized after the merger and is then common knowledge. It does not affect the outsiders' costs.

Demand: We follow the standard assumption that all consumers have the same marginal utility for money, which allows to aggregate their preferences and postulate the existence of a representative consumer. ${ }^{2}$ For $k=1$ to $n$, let $p_{k}$ denote the price of variant $k$, $\mathbf{p}=\left(p_{1}, \ldots, p_{n}\right)^{\prime}$ the column vector of all prices, $x_{k} \geq 0$ the quantity chosen by the consumers, and $\mathbf{x}$ the vector of all quantities. Consumers' surplus is:

\footnotetext{
${ }^{1}$ Some results hold with multidimensional gains. See Remark 2 in Section 3.

${ }^{2}$ See Blackorby et al. (1978)
} 


$$
V(\mathbf{p})=\max _{\mathbf{x}}\left[U(\mathbf{x})-\mathbf{p}^{\prime} \mathbf{x}\right]
$$

The utility function $U$ does not have to be symmetric in $x_{1}, \ldots, x_{n}$. The functions $U$ and $V$ are assumed to be twice differentiable.

Remark 1. The surplus $V$ being the maximum of affine functions of the price vector $\mathbf{p}$ is convex in $\mathbf{p}$.

Moreover, standard duality results in convex analysis (see Rockafellar (1996)) show that, given a convex indirect utility function $V(p)$, there exists a (unique) concave direct utility $U(x)$ satisfying (2). It follows that the convexity of $V$ is necessary and sufficient for consistency with a well-posed consumer's problem. In the remainder of the article, we work with $V$ rather than with $U$.

The demand functions follow from Roy's identity:

$$
D^{k}(\mathbf{p})=-\frac{\partial V}{\partial p_{k}}(\mathbf{p})=-V_{p_{k}}, \quad k=1, \ldots, n
$$

or in matrix notation: $\mathbf{D}=-\mathbf{V}_{\mathbf{p}}$, where $\mathbf{D}=\left(D^{1}, D^{2}, \ldots, D^{n}\right)^{\prime}$. Throughout, we will use a subscript to denote differentiation with respect to prices.

After the merger, the profit function of the merged entity is

$$
\Pi^{I}(\mathbf{p})=\sum_{i \in I}\left(p_{i}-c_{i}(\gamma)\right) D^{i}(\mathbf{p})=\left(\mathbf{p}^{\mathbf{I}}-\mathbf{c}^{\mathbf{I}}(\gamma)\right)^{\prime} \mathbf{D}^{\mathbf{I}}=\left(\mathbf{m}^{\mathbf{I}}\right)^{\prime} \mathbf{D}^{\mathbf{I}}
$$

where $\mathbf{m}^{\mathbf{I}}$ denotes the vector of margins for the products owned by the merged firm. Using similar notations, the profit function of an outsider $J$ writes:

$$
\Pi^{J}(\mathbf{p})=\sum_{j \in J}\left(p_{j}-c_{j}\right) D^{j}(\mathbf{p})=\left(\mathbf{p}^{\mathbf{J}}-\mathbf{c}^{\mathbf{J}}\right)^{\prime} \mathbf{D}^{\mathbf{J}}=\left(\mathbf{m}^{\mathbf{J}}\right)^{\prime} \mathbf{D}^{\mathbf{J}}, \quad J=J_{1}, \ldots, J_{r} .
$$

Oligopoly game: Except in Section 6, firms compete in prices with differentiated products. They choose simultaneously the prices of their brands. A similar situation has been analyzed in Deneckere and Davidson (1985), who, however, mainly focused on the profitability of the merger.

Throughout the paper, we assume that, before and after the merger, the Nash equilibrium exists and is unique. As our focus is not on entry/exit decisions, ${ }^{3}$ we restrict our attention to interior Nash equilibria: demand for each good is positive in equilibrium. In all specific examples under consideration, we provide sufficient conditions for these properties to hold.

\footnotetext{
${ }^{3}$ Werden and Froeb (1998) and Spector (2003) analyze the effects of mergers taking into account entry considerations.
} 
Objective function: We use $\mathbf{p}(\gamma)=\left(p_{1}(\gamma), \ldots, p_{n}(\gamma)\right)^{\prime}$ and $S(\gamma)$ to denote the prices and the consumers' surplus, viewed in (post-merger) equilibrium as functions of $\gamma$ :

$$
S(\gamma)=V(\mathbf{p}(\gamma))
$$

Society's objective, also seen as a function of the magnitude of efficiency gains, is a weighted sum of consumers' surplus and firms' profits:

$$
W_{\alpha}(\gamma)=\alpha S+(1-\alpha) \sum_{K} \Pi^{K}
$$

with $K=I, J_{1}, \ldots, J_{r}$ and $0 \leq \alpha \leq 1$. It is often assumed that antitrust authorities put more weight on consumers' surplus than on firms' profits. (Theoretical foundations can be found in Besanko and Spulber (1993) and Neven and Röller (2005).) Here, however, we do not a priori restrict to $\alpha \geq 1 / 2$.

Existence of a critical threshold: Given a weight $\alpha$, it is natural to ask whether there exists a critical threshold $0<\hat{\gamma}_{\alpha} \leq 1$ such that

$$
W_{\alpha}\left(\hat{\gamma}_{\alpha}\right)=W_{\alpha}^{\text {pre }}
$$

where $W_{\alpha}^{\text {pre }}$ denotes the value of the weighted welfare at the pre-merger Nash equilibrium. Empirical studies often contain estimates of such thresholds. For example, Nevo (2000), Table 7 page 414, estimates $\hat{\gamma}_{1 / 2}$ for a number of actual and hypothetical mergers. See also Werden (1996), who expresses $\hat{\gamma}_{1}$ as a function of diversion ratios. In Section 7, we elaborate on the use of econometric studies for merger assessment when efficiency gains are uncertain.

In theory, the existence of the threshold $\hat{\gamma}_{\alpha}$ does not follow from simple general assumptions. The existence would be guaranteed if it were true that (i) a merger without efficiency gains always reduces the objective: $W_{\alpha}(0)<W_{\alpha}^{\text {pre }}$, (ii) the objective $W_{\alpha}$ always increases in $\gamma$ and (iii) $100 \%$ gains are always sufficient to raise the objective: $W_{\alpha}(1)>W_{\alpha}^{\text {pre }}$. Unfortunately, these properties do not hold in general.

First, when firms compete in price and varieties are strategic complements, it is indeed true that a merger without efficiency gains raises prices, reduces consumers' surplus and increases the profits of the merged entity and of each outsider (unilateral effects). In our notations: $W_{1}(0)<W_{1}^{\text {pre }}$. This property does not hold, however, for total welfare $(\alpha=1 / 2)$. Even when goods are strategic complements, a merger without efficiency gains can raise total welfare (see Appendix A.1). Second, the monotonicity of the objective function $W_{\alpha}$ is not guaranteed. Appendix A.3 shows that the total welfare can even decrease with efficiency gains. Third, $100 \%$ gains are sometimes not sufficient to offset the unilateral effects. This happens, for instance, with zero (or very small) marginal costs. The Logit case studied in Section 4 yields a less obvious example.

If a threshold $\hat{\gamma}_{\alpha}$ does not exist, the tradeoff is trivial: irrespective of the magnitude of the efficiency gains, the merger is either beneficial or detrimental. We concentrate on the 
only case of interest in which $\hat{\gamma}_{\alpha}$ exists. In the following sections, we discuss the existence of $\hat{\gamma}_{\alpha}$ in each specific situations under consideration. For deterministic efficiency gains, say $\gamma=\bar{\gamma}$, the assessment of the merger is straightforward. If $\hat{\gamma}_{\alpha}>\bar{\gamma}$, the merger is blocked, while if $\bar{\gamma}>\hat{\gamma}_{\alpha}$, the merger is welcomed.

Decision under uncertainty: The article investigates the role of uncertainty of efficiency gains in the tradeoff between unilateral effect and efficiency gains. We assume that society is risk neutral with respect to consumers' surplus and firms' profit, i.e. it maximizes $\mathbb{E}_{\gamma}\left(W_{\alpha}\right)$.

Suppose antitrust authorities only know the average value of $\gamma$, and make their decision based only on the comparison of $\hat{\gamma}_{\alpha}$ with $\mathbb{E} \gamma$. When the objective is globally convex or concave, ${ }^{4}$ such a decision rule may lead to the rejection (resp. acceptation) of welfare enhancing (resp. decreasing) mergers, i.e. to type I (resp. II) errors, as shown in Table 1.

Table 1: Merger assessment under limited information

\begin{tabular}{c}
$W_{\alpha}$ is globally \\
\hline convex in $\gamma \quad$ concave in $\gamma$
\end{tabular}

\begin{tabular}{lcc}
\hline \hline Merger is blocked $\left(\hat{\gamma}_{\alpha}>\mathbb{E} \gamma\right)$ & Type I errors may occur & No error \\
Merger is cleared $\left(\hat{\gamma}_{\alpha}<\mathbb{E} \gamma\right)$ & No error & Type II errors may occur \\
\hline
\end{tabular}

Of course, the relevant comparison is between $\hat{\gamma}_{\alpha}$ and $\gamma_{\alpha}^{\mathrm{CE}}$, where $\gamma_{\alpha}^{\mathrm{CE}}$ denotes the certainty equivalent of the lottery $\gamma$ for $W_{\alpha}($.$) , that is, W_{\alpha}\left(\gamma_{\alpha}^{\mathrm{CE}}\right)=\mathbb{E}_{\gamma}\left(W_{\alpha}\right)$. If $\hat{\gamma}_{\alpha}<\gamma_{\alpha}^{\mathrm{CE}}$, the merger enhances welfare, while if $\hat{\gamma}_{\alpha}>\gamma_{\alpha}^{\mathrm{CE}}$, welfare is reduced. The computation of $\gamma_{\alpha}^{\mathrm{CE}}$ would, however, require the knowledge of the entire distribution of $\gamma$.

A preliminary result on the consumers' surplus: For any function $u$ of $\gamma$, we use $\dot{u}$ and $\ddot{u}$ to note its first and second derivatives with respect to $\gamma$. For instance, the vector $\dot{\mathbf{c}}$ measures efficiency gains (see Equation (1)), its $k$ th component is $-\lambda_{k} c_{k}$ if $k \in I$ and zero otherwise.

The first and second derivatives of the consumers' surplus write respectively

$$
\dot{S}=-\sum \dot{p}_{k}(\gamma) D^{k}=-\mathbf{D}^{\prime} \dot{\mathbf{p}}=\left(\mathbf{V}_{\mathbf{p}}\right)^{\prime} \dot{\mathbf{p}}
$$

\footnotetext{
${ }^{4}$ In general, only local properties can be derived. Noting with $\bar{\gamma}$ the mean value and $\sigma^{2}$ the variance of $\gamma$, it is readily confirmed that

$$
\mathbb{E} W_{\alpha}(\gamma)-W_{\alpha}(\bar{\gamma}) \sim \frac{1}{2} \ddot{W}_{\alpha}(\bar{\gamma}) \sigma^{2}
$$

as $\sigma^{2}$ goes to zero. In particular, consider a merger such that $W_{\alpha}(\bar{\gamma})=W_{\alpha}^{\text {pre }}$ and $\sigma^{2}$ is small. The merger should be accepted if $\ddot{W}_{\alpha}(\bar{\gamma})>0$, rejected if $\ddot{W}_{\alpha}(\bar{\gamma})<0$.
} 
and

$$
\ddot{S}(\gamma)=\sum_{k \in N} \sum_{h \in N} \dot{p}_{k} \dot{p}_{h} \frac{-\partial D^{k}}{\partial p_{h}}-\sum_{k \in N} \ddot{p}_{k}(\gamma) D^{k}=\dot{\mathbf{p}}^{\prime} \mathbf{V}_{\mathbf{p p}} \dot{\mathbf{p}}-\mathbf{D}^{\prime} \ddot{\mathbf{p}}
$$

Due to the convexity of $V$ with respect to prices, the term $\dot{\mathbf{p}}^{\prime} \mathbf{V}_{\mathbf{p p}} \dot{\mathbf{p}}$ is nonnegative, while the sign of $\mathbf{D}^{\prime} \ddot{\mathbf{p}}$ is indeterminate. In general, therefore, one cannot sign $\ddot{S}$ nor $\ddot{W}$. In the remainder of the paper, however, we exhibit a number of cases where the first effect dominates.

\section{Linear demand system}

The linear demand system is widely used to model price competition. Theorists refer to it when they need a closed form equilibrium. Empiricists also use it to estimate demand (see, for instance, Pinkse and Slade (2004)). Here we use the most general version of the linear demand model. We impose the minimal restrictions necessary to ensure the strategic complementarity and the existence and uniqueness of a Nash equilibrium. Unlike earlier works (Deneckere and Davidson (1985) and Section 5.4 of Motta (2004)), we do not limit our analysis to symmetric costs or demand systems. In particular, we do not restrict to Bertrand-Shubik demands (see Shubik and Levitan (1980)).

The consumers' surplus rewrites as

$$
V(\mathbf{p})=-\mathbf{A}^{\prime} \mathbf{p}-\frac{1}{2} \mathbf{p}^{\prime} \mathbf{B} \mathbf{p}
$$

where $\mathbf{A}$ is a positive (constant) vector of size $n$ and $\mathbf{B}=\left(b_{h j}\right)_{h, k \in N}$ is a (constant) matrix of size $n$.

According to Remark 1, the matrix $\mathbf{B}$ must be symmetric negative semi-definite. In what follows, we make the additional assumption that $\mathbf{B}$ is nonsingular. These conditions are satisfied when $\mathbf{B}$ is a symmetric diagonally dominant matrix with: $b_{h h}<0$ and $\sum_{k \neq h}\left|b_{h k}\right|<$ $\left|b_{h h}\right|$ for all $h=1$ to $n$ (see Jacob (1990), pp. 240-241). These inequalities imply that if the prices of all varieties increase by the same amount, then all demands decrease. We do not, however, restrict the analysis to this particular case. Throughout, we shall rely only on $\mathbf{B}$ being symmetric negative definite. The demand function, given by Roy's identity, is

$$
\mathbf{D}(\mathbf{p})=-\mathbf{V}_{\mathbf{p}}=\left(\mathbf{D}^{\mathbf{I}}, \mathbf{D}^{\mathbf{J}_{1}}, \ldots, \mathbf{D}^{\mathbf{J}_{\mathbf{r}}}\right)^{\prime}=\mathbf{A}+\mathbf{B} \mathbf{p}
$$

Let $\mathbf{D}_{\mathbf{K}}^{\mathbf{K}}$ denote the submatrix $\left(b_{h k}\right)_{h, k \in K}$. After the merger, the first order conditions for firm $K=I, J_{1}, \ldots, J_{r}$ write

$$
\mathbf{D}^{\mathbf{K}}+\mathbf{D}_{\mathbf{K}}^{\mathbf{K}} \mathbf{m}^{\mathbf{K}}=0
$$

Since the matrices $\mathbf{D}_{\mathbf{K}}^{\mathbf{K}}$ are definite negative, the second-order conditions of the firms' maximization problems are satisfied. In Appendix B.1, we solve for the best reply maps, which are implicitly given by (4), and for the equilibrium prices. Next, we turn to the main result of this section. 
Proposition 1. When the demand system is linear in prices, all equilibrium prices are linear functions of $\gamma$ (that is, $\ddot{\mathbf{p}}=0$ ) and, therefore, consumers' surplus is strictly convex in $\gamma$. In addition, all profit functions (that is, of the merged entity as well as of each outsider) are convex in $\gamma$. As a result, $W_{\alpha}$ is strictly convex in $\gamma$.

Proof. If (for all $h, k) D^{h}$ is linear in $p_{k}$, then the first order condition system is linear in prices and in $\gamma$. Therefore the equilibrium price is linear in $\gamma$. (Its expression is given by (17) in Appendix B.1.) Equation (3) and $\ddot{\mathbf{p}}=0$ yield: $\ddot{S}=(\dot{\mathbf{p}})^{\prime} \mathbf{V}_{\mathbf{p p}} \dot{\mathbf{p}}$. Since $\dot{\mathbf{p}}$ is not zero ${ }^{5}$ and $\mathbf{V}_{\mathbf{p p}}=-B$ is symmetric positive definite, we have: $\ddot{S}>0$.

Next, we prove the convexity of the profit functions. Using the first order conditions (4), the equilibrium value of the profit of firm $K$ writes:

$$
\Pi^{K}=\left(\mathbf{m}^{\mathbf{K}}\right)^{\prime} \mathbf{D}^{\mathbf{K}}=\left(\mathbf{m}^{\mathbf{K}}\right)^{\prime}\left(-\mathbf{D}_{\mathbf{K}}^{\mathbf{K}}\right) \mathbf{m}^{\mathbf{K}} .
$$

Therefore, using the symmetry of $\mathbf{D}_{\mathbf{K}}^{\mathbf{K}}, \dot{\Pi}^{K}=2\left(\mathbf{m}^{\mathbf{K}}\right)^{\prime}\left(-\mathbf{D}_{\mathbf{K}}^{\mathbf{K}}\right) \dot{\mathbf{m}}^{\mathbf{K}}$ and

$$
\ddot{\Pi}^{K}=2\left(\dot{\mathbf{m}}^{\mathbf{K}}\right)^{\prime}\left(-\mathbf{D}_{\mathbf{K}}^{\mathbf{K}}\right) \dot{\mathbf{m}}^{\mathbf{K}} .
$$

Since the matrix $\left(-\mathbf{D}_{\mathbf{K}}^{\mathbf{K}}\right)$ is symmetric positive, we have that $\ddot{\Pi}^{K} \geq 0$ for all $K$.

Proposition 1 emphasizes that in the linear case the objective function of the antitrust authorities (which can be any combination of consumers' surplus and profits) is convex. Therefore, whenever the linearity assumption is realistic, the authorities should not be afraid of uncertainty. On the contrary, uncertainty should be welcomed and firms' should be encouraged to provide verifiable evidence about the variance of the efficiency gains and not only about its mean value. This result is obtained for any structure of the industry. That is, the merged entity as well as any outsider might control several brands. No symmetry assumption is required.

Proposition 1 does not rely on the assumption that efficiency gains are proportional to a scalar $\gamma$. Indeed, it generalizes to multidimensional efficiency gains, as shown in the following remark (proved in Appendix B.3).

Remark 2. Let $\boldsymbol{\gamma}=\left(\gamma_{1}, \ldots, \gamma_{l}\right)^{\prime}$, and assume that after the merger $c_{i}(\gamma)=\left(1-\gamma_{i}\right) c_{i}$, $i \in$ I. The consumers' surplus and the profit of each firm are convex functions of the efficiency gain vector $\gamma$.

\footnotetext{
${ }^{5}$ See $(18)$ in Appendix B.1
} 
To end this section, we discuss the monotonicity of $W_{\alpha}(\gamma)$. The linearity of demand does not ensure that $W_{\alpha}$ is a increasing function of $\gamma$. Yet, provided that a simple technical condition is satisfied, Lemma 1 (see Appendix B.2 for a proof) shows that $W_{1}$ in increasing. That is, consumers unambiguously benefit from an increase in $\gamma$.

Lemma 1. Suppose that goods are strategic complements and the best reply map is a contraction. Then all equilibrium prices are decreasing with $\gamma(\dot{\mathbf{p}} \leq 0)$. Consumers' surplus is an increasing function of $\gamma$. Outsiders' profits decrease with $\gamma$.

Yet, even under the assumptions of the lemma, the insider's profit may decrease with $\gamma$, as shown by the example detailed in Appendix A.2. Therefore, if a weight large enough is put on firms, $W_{\alpha}$ can be decreasing. Looking more closely at the total welfare $(\alpha=1 / 2)$, we compute:

$$
\begin{aligned}
2 \dot{W}_{1 / 2}=\dot{S}+\sum_{K} \dot{\Pi}^{K} & =-\mathbf{D}^{\prime} \dot{\mathbf{p}}+2 \sum_{K}\left(\mathbf{D}^{\mathbf{K}}\right)^{\prime} \dot{\mathbf{m}}^{\mathbf{K}} \\
& =-\left(\mathbf{D}^{\mathbf{I}}\right)^{\prime} \dot{\mathbf{p}}^{\mathbf{I}}+2\left(\mathbf{D}^{\mathbf{I}}\right)^{\prime} \dot{\mathbf{m}}^{\mathbf{I}}+\sum_{J \neq I}\left(\mathbf{D}^{\mathbf{J}}\right)^{\prime} \dot{\mathbf{p}}^{\mathbf{J}} .
\end{aligned}
$$

Under the assumptions of the Lemma 1, the last term in (7) is negative: as far as the goods controlled by the outsiders are concerned, outsiders lose more than consumers gain from a higher efficiency of the new entity. The first term being always positive, ${ }^{6}$ the sign of $\dot{W}_{1 / 2}$ is indeterminate. Intuitively, total welfare should increase with efficiency gains. Appendix A.3 shows that this is not true, even when the products are strategic complements and the best reply map is a contraction.

The linearity of demand is, therefore, not sufficient to rule out counter-intuitive situations. In contrast to the surprising properties of the first derivatives, Proposition 1 yields clear-cut results for the curvature of consumers' surplus and profits. The linearity of demand implies that competition authorities should love the risk related to the magnitude of efficiency gains (irrespective of the relative weights assigned to consumers and firms). Next, we turn to more general demand functions.

\section{Mergers to monopoly}

In this section, we examine the attitude towards risk for mergers that lead to a monopoly $(I=N)$, that is, the case where unilateral effects are expected to be the strongest. On the contrary, Section 5 posits Bertrand competition with homogenous products before and after the merger, that is, the weakest unilateral effects. Both sections can thus be thought of as complementary. These restrictions allow to carry out the analysis for general demand functions.

\footnotetext{
${ }^{6}$ The middle term, which is the derivative of the insider's profit, can be negative, as shown in Appen$\operatorname{dix}$ A.2.
} 
Lemma 2 (Convexity of monopoly profits). The equilibrium monopoly profit is a convex function of the magnitude of the efficiency gains.

Proof. The monopoly's profit is $\Pi^{I}(\gamma)=\max _{\mathbf{p}}\left[(\mathbf{p}-\mathbf{c}(\gamma))^{\prime} \mathbf{D}(\mathbf{p})\right]$. As $\mathbf{c}(\gamma)$ is an affine function of $\gamma, \Pi^{I}(\gamma)$ is convex in $\gamma$ as a maximum of affine functions.

Lemma 2 shows that for any demand functions, the monopoly's profit is convex in $\gamma$. That is, firms are willing to take risks to create a monopoly. ${ }^{7}$ For a given mean, say zero, they are ready to accept a large variance.

To derive specific results on consumers' surplus for general demand functions, we postulate a symmetric environnement.

Assumption 1 (Symmetry). Before and after the merger, consumers perceive brands symmetrically. All marginal costs of production are identical.

Given the symmetry of demand functions in $p_{1}, \ldots, p_{n}$, it is readily confirmed that at the optimum: $p_{1}=\ldots=p_{n}$. Therefore there is no loss of generality in assuming that the merged entity maximizes its profit under the constraint that $p_{1}=\ldots=p_{n}=p$. Let $\Phi$ be the Chamberlin's $D D$ curve, i.e. the demand function for one product when all prices are equal (see e.g. Anderson, de Palma, and Kreider (2001a))

$$
\Phi(p)=D^{i}(p, \ldots, p) .
$$

Then the post-merger profit function is:

$$
\Pi^{I}(p)=n[p-(1-\gamma) c] \Phi(p) .
$$

Let $p(\gamma)$ be the post-merger monopoly price. Hereafter, we continue to note with a dot derivatives with respect to $\gamma($ e.g. $\dot{p})$ and with a prime derivatives w.r.t. the price (e.g. $\left.\Phi^{\prime}\right)$.

Definition 1. Let $\varepsilon(p)=-\frac{p \Phi^{\prime}}{\Phi}$ denote the price elasticity of the demand function $\Phi$ and let $E(p)=\frac{p \Phi^{\prime \prime}}{\Phi^{\prime}}$ denote the price elasticity of $\Phi^{\prime}$ the derivative of the demand function. Finally let $\Psi(p)=E / \varepsilon$ be the ratio of both elasticities.

Appendix C.1 shows that $\Psi(p)$ is nothing but the elasticity of the slope of the inverse demand curve, evaluated at $\Phi(p)$. Appendix C.2 presents the first-order condition of the monopoly problem and checks that the second-order condition is satisfied if and only if $\Psi(p(\gamma))+2>0$. Appendix C.3 computes the second-order derivatives of consumers' surplus and firms profits, and establishes the following result.

\footnotetext{
${ }^{7}$ This is consistent with the high number of mergers that destroy value observed in practice.
} 
Lemma 3. The welfare function $W_{\alpha}$ is strictly convex at $\gamma$ if and only if

$$
\frac{\alpha \Psi^{\prime}}{2+\Psi}+[\alpha+(1-\alpha)(2+\Psi)] \frac{\varepsilon}{p(\gamma)}>0
$$

where $\Psi, \Psi^{\prime}$ and $\varepsilon$ are evaluated at $p(\gamma)$.

Because of the second-order condition, the bracketed term in (8) is strictly positive, which yields Proposition 2.

Proposition 2. If $\Psi$ is nondecreasing with $p$, then, for all $0 \leq \alpha \leq 1$, the objective $W_{\alpha}$ is globally convex in $\gamma$.

Proposition 2 characterizes a large class of demand functions for which $W_{\alpha}$ is globally convex. In consequence, for this class, for a given mean, uncertainty about efficiency gains raises the expected value of $W_{\alpha}$.

As a first illustration of Proposition 2, suppose goods are homogenous and demand is given by $(a-b p)^{\beta}$, with $\beta b>0$. In this case, the function $\Psi$ is constant and equal to $(1-\beta) / \beta$. When $\beta \notin[-1,0]$, the second-order condition $\Psi+2>0$ is satisfied and Proposition 2 applies. Similarly, if $\Phi=A \exp [a-b p](A, b>0)$, then $\Psi=-1$. Next, we present examples with imperfect substitutes. First, we consider constant elasticity of substitution (CES) demand system (see Vives (1999), pages 147-148)

$$
D^{i}\left(p_{1}, \ldots, p_{n}\right)=(\beta \theta)^{1 /(1-\beta \theta)} \frac{p_{i}^{1 /(\beta-1)}}{\left(p_{1}^{\beta /(\beta-1)}+\ldots+p_{n}^{\beta /(\beta-1)}\right)^{(1-\theta) /(1-\beta \theta)}},
$$

with $0<\beta \theta<1, \beta \leq 1$ and $\theta<1$. The elasticity of substitution, defined as the derivative of $\ln \left(D_{i} / D_{j}\right)$ with respect to $\ln \left(p_{i} / p_{j}\right)$, is $1 /(1-\beta)$. For $\beta=1$, goods are homogenous.

Corollary 1 (Constant elasticity of substitution). If the demands $D^{i}$ exhibit a constantelasticity of substitution, then $\Psi^{\prime}=0$, and therefore $W_{\alpha}$ is globally convex in $\gamma$, for all $0 \leq \alpha \leq 1$.

Proof. In that case,

$$
\Phi(p)=n^{\frac{-(1-\theta)}{(1-\beta \theta)}}(\beta \theta)^{1 /(1-\beta \theta)} p^{-1 /(1-\beta \theta)},
$$

whence we deduce that: $\Psi=-2+\beta \theta>-2$. The function $\Psi$ is constant and the secondorder conditions are satisfied. Proposition 2 applies.

Next, we turn to the symmetric Logit demand system with an outside option ${ }^{8}$

$$
D^{i}\left(p_{1}, \ldots, p_{n}\right)=\frac{\exp \left(-p_{i} / \sigma\right)}{\exp \left(-p_{0} / \sigma\right)+\ldots+\exp \left(-p_{n} / \sigma\right)}
$$

\footnotetext{
${ }^{8}$ See Werden and Froeb (1994) for a thorough study of mergers relying on the Logit model.
} 
where $\sigma>0$ and $p_{0}$ is the price of the outside good. The Chamberlin's demand is

$$
\Phi(p)=\frac{\exp (-p / \sigma)}{\exp \left(-p_{0} / \sigma\right)+n \exp (-p / \sigma)} .
$$

A simple change of variables shows that the monopoly price $p(\gamma)$ is such that $p(\gamma) / \sigma$ only depends on $c / \sigma$ and $p_{0} / \sigma$. After the merger, the market share covered by the monopolist is $n \Phi(p(\gamma))$.

Lemma 4. With a Logit symmetric demand, the objective $W_{\alpha}$ is a convex function of $\gamma$ if and only if the market coverage by the monopolist is lower than $1 /(2 \alpha)$.

Proof. It is readily confirmed that

$$
\Psi=n \exp \left[\left(p_{0}-p\right) / \sigma\right]-1, \quad \Psi^{\prime}=(-n / \sigma) \exp \left[\left(p_{0}-p\right) / \sigma\right], \quad \frac{\varepsilon}{p}=\frac{1 / \sigma}{n \exp \left[\left(p_{0}-p\right) / \sigma\right]+1} .
$$

Straightforward computations show that (8) writes: $\exp \left[\left(p-p_{0}\right) / \sigma\right]>n(2 \alpha-1)$, which is equivalent to $n \Phi(p(\gamma))<1 /(2 \alpha)$.

Since the market coverage is lower than $100 \%$, the following corollary obtains.

Corollary 2. Suppose $\alpha \leq 1 / 2$. With a Logit symmetric demand, the objective $W_{\alpha}$ is globally (strictly) convex in $\gamma$.

Corollary 2 implies in particular that the total welfare is globally convex in $\gamma$ (case $\alpha=1 / 2$ ). Next, we analyze the curvature of the consumers' surplus $S$ (case $\alpha=1$ ). Here, we get only local results around $\hat{\gamma}_{1}$. Recall that $\hat{\gamma}_{1}$ is the critical threshold for efficiency gains associated with the consumers' surplus. Appendix C.4 shows that

Corollary 3. With a Logit symmetric demand, the consumers' surplus is locally convex at $\hat{\gamma}_{1}$ if and only if

$$
\frac{p_{0}}{\sigma}-\frac{c}{\sigma} \leq \frac{2 n}{2 n-1}-\ln n
$$

The set of parameters defined by condition (9) must be compared to the admissible region, namely the set of $\left(c / \sigma, p_{0} / \sigma\right)$ for which efficiency gains are potentially able to offset unilateral effects. The admissible region, depicted on Figure 1, is located below the curve $\hat{\gamma}_{1}\left(c / \sigma, p_{0} / \sigma\right)=1$ corresponding to $100 \%$ gains. For efficiency gains to be able to compensate the unilateral effect, the outside good must be sufficiently attractive $\left(p_{0} / \sigma\right.$ not too high) and the potential for cost reduction must be sufficiently high $(c / \sigma$ not too small). The straight line $\ddot{S}\left(\hat{\gamma}_{1}\right)=0$ splits the admissible region in two areas where the 
consumers' surplus is locally concave (resp. convex) at $\hat{\gamma}_{1}{ }^{9}$ This example shows that no general conclusion as to the curvature of the consumers' surplus can be drawn.

Lemma 5. Efficiency gains can offset the unilateral effect if only if

$$
p_{0} \leq p^{+}(c)+\sigma \ln \left[\frac{p^{+}(c)-(\sigma+c)}{(\sigma n+(n-1) c)-(n-1) p^{+}(c)}\right]
$$

where

$$
p^{+}(c)=\frac{\sigma+c}{2}+\sqrt{\left(\frac{\sigma+c}{2}\right)^{2}+\frac{\sigma c}{n-1}}
$$

Proof. See Appendix C.4

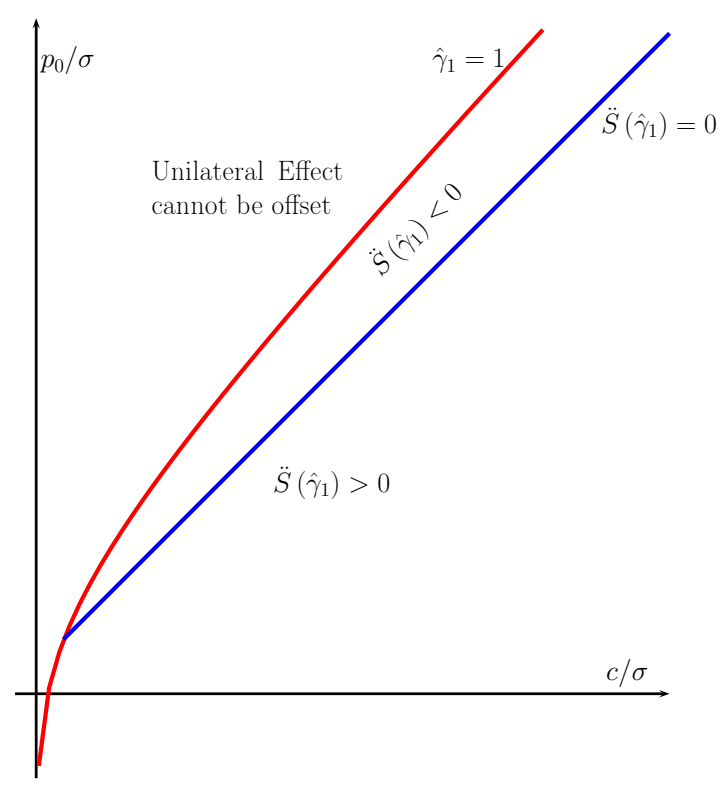

Figure 1: Curvature of consumers' surplus $S$ at $\hat{\gamma}_{1}$ (symmetric Logit demand system, $n=2$ )

\section{$5 \quad$ Mergers without any unilateral effect}

After having investigated mergers leading to monopolies, we turn to situations where, on the contrary, the post-merger environment is highly competitive. To this end, we assume

\footnotetext{
${ }^{9}$ For $n=2$, the curves $\hat{\gamma}_{1}=1$ and $\ddot{S}\left(\hat{\gamma}_{1}\right)=0$ intersect at $c / \sigma=2 / 3$ and $p_{0} / \sigma=2-\ln 2$. As a consequence, for any admissible set of parameter $\left(c, p_{0}, \sigma\right)$ satisfying $p_{0} \leq 1.3 \sigma$, the consumers' surplus is locally convex at $\hat{\gamma}_{1}$.
} 
Bertrand competition with homogeneous goods, the fiercest kind of competition. In particular, if firms share the same technology, prices are driven down to the common marginal cost of production. Yet, thanks to a merger, a firm can obtain some market power if its marginal cost becomes lower than that of its competitors. Since we are interested in post-merger competitive environments, we impose that, after the merger, each firm exerts competitive pressure on the others. That is, we rule out drastic efficiency gains and losses.

Formally, let $c$ be the common pre-merger constant marginal cost. After the merger, the marginal cost of the merged entity amounts to $(1-\gamma) c$ with $\gamma \leq 1$. The marginal cost of the outsider(s) remains at $c$. We note $p^{m}($.$) the (unconstrained) monopoly price as a$ function of the marginal cost: $p^{m}(c)=\arg \max _{p}(p-c) D(p)$ and we adopt the following assumption:

Assumption 2. Efficiency gains and losses are (almost) never drastic, i.e. the inequalities

$$
c \leq p^{m}((1-\gamma) c) \quad \text { and } \quad(1-\gamma) c \leq p^{m}(c)
$$

hold (almost) everywhere on the support of the random variable $\gamma$.

Suppose that $n$ mono-product and symmetric firms initially compete $\grave{a}$ la Bertrand and consider a merger involving $l$ insiders, with $l \leq n-1$. Absent efficiency gain or loss, the consumers' surplus remains unchanged, as well as the industry's profit (still at zero). There is no unilateral effect. In our notations: $\hat{\gamma}_{1}=0$. Moreover, when there are two or more outsiders $(l \leq n-2)$ and as long as Assumption 2 holds, the merger leaves the price unchanged, irrespective of the sign and magnitude of efficiency gains. In particular, efficiency losses, if any, play no role. Society benefits from efficiency gains (which are, however, entirely kept by the merged entity), but cannot suffer from efficiency losses. Things are different when the merger creates an asymmetric duopoly $(l=n-1)$. Here, consumers and society as a whole suffer from efficiency losses. We now concentrate on this case.

Under Assumption 2, all sales are made by the firm with the lowest marginal cost and the post merger equilibrium price is the largest one: $p(\gamma)=\max \{c,(1-\gamma) c\}$. Figure 2 depicts the post-merger firms' profits, consumers' surplus and total welfare $2 W_{1 / 2}$ as functions of $\gamma$. From the expression of $p(\gamma)$, it is transparent that the price cannot decrease and therefore consumers' surplus cannot increase. As soon as there is a positive probability that $\gamma<0$, it is even worse: consumers' surplus decreases in expectation. That is, in this set-up, efficiency gains are never passed on to consumers, but efficiency losses are. Such a merger would not be allowed by antitrust authorities, should they care only for consumers' surplus $(\alpha=1)$. On the other hand, as soon as authorities recognize that firms benefit from the merger $(\alpha<1)$, they face a tradeoff between increased efficiency and creation of market power.

Proposition 3. Under Bertrand competition and Assumption 2, there exists a threshold value $\hat{\alpha} \in(1 / 2,2 / 3]$ such that, for all $\alpha \leq \hat{\alpha}$, any merger creating a duopoly and satisfying 


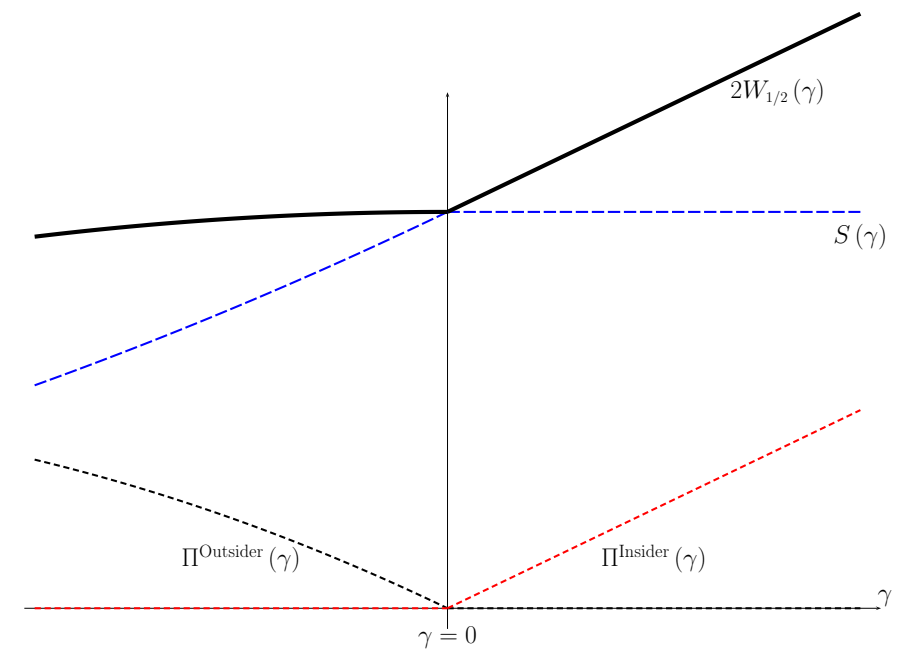

Figure 2: Profits, consumers' surplus and total welfare for a Bertrand duopoly

$\mathbb{E} \gamma=0$ raises the expected value of the objective function $W_{\alpha}$. Formally

$$
\mathbb{E}_{\gamma}\left(W_{\alpha}\right) \geq W_{\alpha}(\mathbb{E} \gamma)=W_{\alpha}(0)=W_{\alpha}^{p r e}
$$

Proposition 3 establishes that if a weight large enough is put on firms and if efficiency gains are zero in expectation $\left(\mathbb{E} \gamma=0=\hat{\gamma}_{1}\right)$, then uncertainty of efficiency gains is welcomed. The proof, in Appendix D, shows that $\hat{\alpha}=\left[D(c)+D\left(p^{m}(c)\right)\right] /\left[2 D(c)+D\left(p^{m}(c)\right)\right]$.

The inequality (12) does not follow from Jensen's lemma since the objective function $W_{\alpha}$ is not globally convex in $\gamma$ (see Figure 2). It may, however, be locally convex around $\hat{\gamma}_{1}=0$. More precisely, Figure 2 shows that the industry's profit $\Pi^{\text {Insider }}+\Pi^{\text {Outsider }}$ has a convex kink at 0 , while the consumers' surplus has a concave kink at 0 . It is easy to check that the right and left derivatives of $W_{\alpha}$ at 0 are $(1-\alpha) c D(c)$ and $(2 \alpha-1) c D(c)$ respectively. Therefore, $W_{\alpha}$ has a convex kink at 0 if and only if $\alpha \leq 2 / 3$. It follows that, for any $\alpha \leq 2 / 3$, it exists $\eta>0$ such that, if the random variable $\gamma$ has zero mean and takes its values in $[-\eta, \eta]$, then the merger raises $\mathbb{E}_{\gamma}\left(W_{\alpha}\right)$. Proposition 3 introduces a threshold $\hat{\alpha} \leq 2 / 3$ such that this "convexity-like" property is valid without any restriction on the support of $\gamma$ (besides Assumption 2).

Finally, note that $\widehat{\alpha}$ is always (strictly) larger than $1 / 2$ : from the ex ante point of view, a merger with zero efficiency gains in expectation raises the standard welfare criterion, which puts equal weights to firms and consumers. 


\section{Mergers under Cournot competition}

In this section, we examine the effects of uncertainty of efficiency gains for mergers when firms compete à la Cournot. Costs are asymmetric and mergers under consideration involve an arbitrary number of parties, $2 \leq l \leq n$. All notations used below have been introduced in Section 2.

After the merger, the $r$ outsiders use the same technology as before, while the merged entity enjoys a marginal cost of $(1-\gamma) c^{I}$, where $c^{I}$ denotes the marginal cost of the merged entity absent efficiency gains $(\gamma=0)$. In the spirit of Farrell and Shapiro (1990), we distinguish cost reductions due to the reallocation of production, which lead to $c^{I}$, from the ones linked to efficiency gains, which lead to $(1-\gamma) c^{I}$. In the Cournot model with constant return to scale, a merger of two (or more) asymmetric firms should, in principle, lead to the closing of the less efficient insider(s). In that case, $c^{I}$ would be equal to the smallest marginal cost among insiders'. Yet, such a rationalization of the production might not be achievable by the merging firms, so we assume $c^{I} \geq \min _{i \in I} c_{i}$ but we do not a priori impose equality.

The inverse demand curve is denoted $P(Q)$, where $Q$ is the sum of the quantities produced by all firms. Let $Q(\gamma)$ denote the equilibrium quantity after the merger. From now on, a prime is used for derivatives with respect to quantity (e.g. $P^{\prime}$ ), while the dot still indicates a derivative w.r.t. $\gamma($ e.g. $\dot{Q})$. The elasticity $\Theta(Q)=Q P^{\prime \prime}(Q) / P^{\prime}(Q)$ of the slope of the inverse demand function plays a crucial role in the analysis. When evaluated at $Q=\Phi(p)$, it corresponds to the function $\Psi(p)$ used in Section 4. The assumption $\Theta+2>0$ ensures that the maximization problem of each firm is concave (for any configuration of the industry). By adding the f.o.c. of each firm, we check that $Q(\gamma)$ is the unique solution to

$$
(r+1) P(Q)+Q P^{\prime}(Q)=(1-\gamma) c^{I}+\sum_{j \in J} c_{j},
$$

where $J$ denotes the set of outsiders. Lemma 6 provides a necessary and sufficient condition for efficiency gains to be able to compensate unilateral effects. This condition only depends on the pre-merger price and on the sum of the marginal costs of the insiders before the merger.

Lemma 6 (Farrell and Shapiro (1990), Proposition 1). Let $p^{\text {pre }}$ denote the pre-merger equilibrium price. Efficiency gains are able to offset the unilateral effects of the merger (from consumers' point of view) if and only if

$$
\sum_{i \in I} \frac{p^{\text {pre }}-c_{i}}{p^{\text {pre }}} \leq 1 .
$$

Proof. The pre-merger equilibrium quantity $Q^{\text {pre }}$ is solution to $n P(Q)+Q P^{\prime}(Q)=\sum_{k \in N} c_{k}$. Noting $p^{\text {pre }}=P\left(Q^{\text {pre }}\right)$ the pre-merger price, we get: $Q^{\text {pre }} P^{\prime}\left(Q^{\text {pre }}\right)=\sum_{k \in N} c_{k}-n p^{\text {pre }}$. Us- 
ing (13), it is straightforward that the price after the merger is exactly equal to $p^{\text {pre }}$ if and only if $c^{I} \gamma=(l-1) p^{\text {pre }}-\sum_{i \in I} c_{i}+c^{I}$. The condition $\gamma \leq 1$ leads to (14).

For the inequality (14) to hold, insiders should not be too efficient ${ }^{10}$ before the merger. Absent efficiency gains, the merger harms consumers (See Farrell and Shapiro (1990), Proposition 2). That is, the merger is harmful (due to unilateral effects) but if (14) holds, amendable (thanks to efficiency gains). The critical threshold $\hat{\gamma}_{1}$ is, then, given by $c^{I} \hat{\gamma}_{1}=-\left(p^{\text {pre }}-c^{I}\right)+\sum_{i \in I} p^{\text {pre }}-c_{i}$.

The effect of the uncertainty about $\gamma$ on the expected value of the consumers' surplus remains linked to the curvature of $S$ with respect to $\gamma$.

Lemma 7. The consumers' surplus is locally convex at $\gamma$ if and only if

$$
1-\frac{Q \Theta^{\prime}}{r+2+\Theta}>0
$$

Lemma 7, proved in Appendix E, parallels Lemma 3 of Section 4. Both results coincide exactly when we look at the consumers' surplus for mergers that create a monopoly (replace $r$ by 0 in Equation (15) and $\alpha$ by 1 in Equation (8)). This similarity is not coincidental, as a monopoly selling an homogeneous good can equivalently maximize in price or quantity. Recall, however, that in this section (contrary to Section 4), firms do not share the same technology and the mergers under consideration do not necessarily create a monopoly.

Proposition 4. If $\Theta$ is non increasing in $Q$ and larger than -2, then the consumers' surplus is globally convex in $\gamma(\ddot{S}>0)$.

Proposition 4, which follow directly from condition (15), applies to a large class of demand functions. A subclass of interest consists of demand functions such that the function $\Theta$ is constant and larger than -2 (to ensure that the second-order conditions hold). This family is described by three parameters:

$$
P(Q)=\max \left\{a+\frac{b}{1+\theta} Q^{1+\theta} ; 0\right\}
$$

with $-2<\theta \neq-1$ and $b<0$. If $\theta>-1$, the intercept $a$ must be positive to get a positive demand. If $\theta<-1, a$ must be negative to guarantee that the demand tends to zero as the price goes to infinity. For $\theta=-1$, we define

$$
P(Q)=\max \{a+b \log (Q) ; 0\},
$$

with $a>0$ and $b<0$. This family contains demands with constant elasticity, $P(Q)=$ $a-b \varepsilon Q^{-1 / \varepsilon}$, with $\varepsilon>1($ take $\theta=-(1+1 / \varepsilon))$. For all demands in this family, the function

\footnotetext{
${ }^{10}$ In particular, if all marginal costs equal $c$, then (14) writes $\frac{p^{\text {pre }}-c}{p^{\text {pre }}} \leq 1 / l$. If, in addition, demand is linear $(P=a-b Q)$, then (14) becomes $c \geq(l-1) a /(n+l)$, which defines a lower bound for $c$.
} 
$\Theta$ is equal to the constant $\theta$ and Proposition 4 applies. Another particular case encompassed in this family is the linear demand $(\theta=0)$ for which the curvature of the profit functions can also be determined.

Lemma 8. If demand is linear, $P=a-b Q$, with $a, b>0$, then, for all $0 \leq \alpha \leq 1$, the objective function $W_{\alpha}$ is globally convex in $\gamma$.

Proof. The equilibrium profit of any firm writes $\Pi^{k}=b\left(q^{k}\right)^{2}$ where $q^{k}$ is linear w.r.t. marginal costs. Therefore, all profit functions are convex in $\gamma$. Proposition 4 shows that $S$ is convex, therefore $W_{\alpha}$ is convex as a combination of convex functions.

To summarize, in the Cournot environment with asymmetric costs and an arbitrary number of outsiders, the post-merger consumers' surplus appears to be a convex function of the efficiency gains for a large family of demand functions. The curvature of the profits function is harder to characterize. For linear demand, the convexity of profits hold.

\section{Lessons for merger control}

The analysis of horizontal mergers hinges on a tradeoff between unilateral effects and efficiency gains. This article examines the role of uncertainty (on efficiency gains) in such a tradeoff. Common wisdom is that antitrust authorities should be very cautious about random gains. Our results show that dismissing efficiency gains on the sole ground that they are uncertain would not be theoretically founded. Indeed, the attitude towards uncertainty depends on the curvature of the social objective function. We have exhibited a number of situations where the objective is convex in the efficiency gains, implying that competition authorities should welcome the risk for a given expectation of efficiency gains.

In particular, we have shown that the linearity of demand ensures the convexity of profits and consumers' surplus in an otherwise general price competition setup. When mergers create a monopoly, the profit is always convex in the efficiency gains; the consumers surplus is convex for CES demand systems; total welfare is convex for a Logit demand. When the merger creates a duopoly and goods are homogenous, a convexity-like result holds for total welfare (for any demand function). When firms compete in quantity with asymmetric costs, the consumers' surplus is convex in efficiency gains for a large class of demand functions. This is not to say that the convexity property is general. The Logit example shows that, for a merger to monopoly, the consumers' surplus can be locally convex or concave, depending on the precise value of the underlying structural parameters.

The article provides some guidance for empirical merger analysis. Our results imply that functional specifications may entail implicit restrictions on the attitude towards risk regarding the magnitude of efficiency gains. When two different demand systems fit the data equally well, welfare could be convex in one case but concave in the other. In the 
former case, it is more likely that efficiency gains are found to compensate the unilateral effect than in the latter case.

Merging parties commonly provide authorities with econometric studies tending to show that the unilateral effects of the merger are weak and will be offset by modest efficiency gains. Typically, these studies estimate structural oligopolistic models and use these estimations to simulate the post-merger market equilibrium under various assumptions. A huge body of empirical literature has grown, estimating unilateral effects for mergers in various industries: see, among others, Nevo (2000) in the U.S. ready-to-eat cereals industry, Focarelli and Panetta (2003) in the Italian bank sector, Pinkse and Slade (2004) in the U.K. beer industry, and Ivaldi and Verboven (2005) for the European truck industry. These studies allow to estimate critical thresholds for the magnitude of efficiency gains, i.e. lower bounds for gains to offset unilateral effects.

Empirical studies, of course, account for uncertainty in the environment, by using error terms in the demand or cost equations. Note, however, that the uncertainty about efficiency gains is of a different nature than that of demand. Efficiency gains affect future costs and are therefore impossible or extremely difficult to estimate ex ante. Empirical studies usually provide point estimate for the threshold above which efficiency gains offset unilateral effect. When the social objective is convex in the efficiency gains and they are not certain, a point estimate of the critical threshold overestimates the necessary gains and might lead to block welfare-enhancing mergers (type I errors). ${ }^{11}$ On the other hand, when the social objective is concave the threshold is underestimated and welfare-deteriorating mergers might be authorized (type II errors). Our results suggest that presenting the objective as a function of future costs might yield a more accurate idea of the market after the merger.

It would be of interest to extend our analysis to situations where the merging firms do not know the exact value of the post-merger cost, but have a better information about its distribution than the authorities (in the spirit of Besanko and Spulber (1993) or Lagerlöf and Heidhues (2005)). Our results might help to better understand the information disclosure issue in such cases. Although this article focuses on horizontal mergers, the argument applies to any situations with a market power - efficiency tradeoff. Williamson (1968) points dissolution, vertical and conglomerate mergers. Many other practices under the scrutiny of antitrust authorities (e.g. joint-ventures, collusion, bundling) give rise to such tradeoffs. In all these cases, the curvature of the social objective plays a crucial role whenever efficiencies are uncertain.

\footnotetext{
${ }^{11}$ For instance, Pinkse and Slade (2004) use a linear demand system to evaluate the impact of mergers in the U.K. brewing industry. They write page 641 that "the costs of the merging firms would have to fall by about $20 \%$ to just offset the increase in market power." They conclude that "a reduction of the required magnitude would not have been possible". Their calculation is done under the implicit assumption that $\gamma$ is perfectly known. Under the chosen specification, we have seen that consumers' surplus is convex. If they had allowed $\gamma$ to be distributed over an interval, the convexity of $S$ would have implied a requirement for the expectation of efficiency gains lower than $20 \%$.
} 


\section{References}

Simon P. Anderson, André de Palma, and Brent Kreider. Tax incidence in differentiated product oligopoly. Journal of Public Economics, 81(2):173-192, August 2001a.

David Besanko and Daniel F. Spulber. Contested mergers and equilibrium antitrust policy. Journal of Law Economics \& Organization, 9(1):1-29, April 1993.

Raymond Deneckere and Carl Davidson. Incentives to form coalitions with bertrand competition. RAND Journal of Economics, 16(4):473-86, Winter 1985.

Joseph Farrell and Carl Shapiro. Horizontal mergers: An equilibrium analysis. American Economic Review, 80(1):107-26, March 1990.

Dario Focarelli and Fabio Panetta. Are mergers beneficial to consumers? Evidence from the market of bank deposits. American Economic Review, 93(4):1152-1172, September 2003.

Marc Ivaldi and Frank Verboven. Quantifying the effects from horizontal mergers in european competition policy. International Journal of Industrial Organization, 23(9-10): 669-691, December 2005.

Bill Jacob. Linear Algebra. W.H. Freeman and Company, New York, U.S.A., 1990.

Johan N.M. Lagerlöf and Paul Heidhues. On the desirability of an efficiency defense in merger control. International Journal of Industrial Organization, 23(9-10):803-827, December 2005.

Massimo Motta. Competition policy. Cambridge University Press, Cambridge, U.K., 2004.

Damien J. Neven and Lars-Hendrick Röller. Consumer surplus vs. welfare standard in a political economy model of merger control. International Journal of Industrial Organization, 23(9-10):829-848, December 2005.

Aviv Nevo. Mergers with differentiated products: The case of the ready-to-eat cereal industry. RAND Journal of Economics, 31(3):395-421, Autumn 2000.

Joris Pinkse and Margaret E. Slade. Mergers, brand competition, and the price of a pint. European Economic Review, 48(3):617-643, June 2004.

Ralph Tyrell Rockafellar. Convex Analysis. Princeton University Press, Princeton, U.S.A., 1996.

Martin Shubik and Richard Levitan. Market Structure and Behavior. Harvard University Press, Cambridge, MA, 1980.

David Spector. Horizontal mergers, entry, and efficiency defences. International Journal of Industrial Organization, 21(10):1591-1600, December 2003. 
Xavier Vives. Oligopoly Pricing: Old ideas and new tools. MIT Press, Cambridge and London, 1999.

Gregory J. Werden. A robust test for consumer welfare enhancing mergers among sellers of differentiated products. Journal of Industrial Economics, 44(4):409-413, December 1996.

Gregory J. Werden and Luke M. Froeb. The effects of mergers in differentiated products industries: Logit demand and merger policy. Journal of Law Economics $E$ S Organization, 10(2):407-426, October 1994.

Gregory J. Werden and Luke M. Froeb. The entry-inducing effects of horizontal mergers: An exploratory analysis. Journal of Industrial Economics, 46(4):525-43, December 1998.

Oliver Williamson. Economies as an antitrust defense: The welfare tradeoffs. American Economic Review, 58(1):18-36, March 1968.

\section{Appendix}

\section{A Some properties of mergers under price competition}

In this Appendix, we present three counter-intuitive properties of mergers when firms compete in prices. The examples below rely on linear demand systems, which are analyzed systematically in Section 3 and Appendix B. For all examples, we have checked that (i) the demand is consistent with a well-posed consumer's problem (see Remark 1), (ii) there exists a unique Nash equilibrium, (iii) all varieties are produced at the pre- and post-merger equilibria, (iv) the varieties are strategic complements and (v) the best reply map is a contraction. (See Lemma 1, Appendix B and Footnote 15 for more details.)

\section{A.1 A merger without efficiency gains can raise total welfare}

When firms compete in price and varieties are strategic substitutes, it is well-known that a merger can reduce price and raise total welfare. ${ }^{12}$ We show in this section that this may also happen for substitute goods under strategic complementary. The intuition goes as follows. A merger might involve relatively inefficient firms whose goods are (relatively) not valued much by consumers and (relatively) distinct from outsiders' goods. As a result of the merger, all prices increase but the outsiders' prices increase less than insiders' ones. Therefore, while the quantities produced by the insiders decrease, some of the quantities

\footnotetext{
${ }^{12}$ For instance, two firms selling complementary goods exert a negative price externality on each other. If they merge, they reduce prices and at the same time earn more profit. This property is known as the Cournot effect.
} 
produced by the outsiders might increase. If one of the outsider is very efficient, the merger shifts production from inefficient firms to an efficient one, which reduces total costs of production. This gain might be greater than consumers' loss due to higher prices.

To illustrate, take $n=3$ monoproduct firms and consider the following demand system:

$$
\left\{\begin{array}{l}
D^{1}=10-p_{1}+\frac{1}{2} p_{2}+\frac{1}{20} p_{3} \\
D^{2}=10+\frac{1}{2} p_{1}-p_{2}+\frac{1}{20} p_{3} \\
D^{3}=10,000+\frac{1}{20} p_{1}+\frac{1}{20} p_{2}-p_{3}
\end{array}\right.
$$

Assume that the marginal costs of production are

$$
c_{1}=c_{2}=9 \text { and } c_{3}=0 .
$$

The pre-merger Nash equilibrium is:

$$
\mathbf{p}^{\text {pre }}=\left(\begin{array}{c}
179.633 \\
179.633 \\
5008.98
\end{array}\right), \mathbf{D}^{\text {pre }}=\left(\begin{array}{c}
170.633 \\
170.633 \\
5008.98
\end{array}\right), \mathbf{\Pi}^{\text {pre }}=\left(\begin{array}{c}
29,115.5 \\
29,115.5 \\
25,089,897.0
\end{array}\right) .
$$

Consumers' surplus is $S^{\text {pre }}=-37,622,304.2$, while total welfare is $W_{1 / 2}^{\text {pre }}=-12,474,176.1$. Suppose that firms 1 and 2 merge without generating any efficiency gains. The post-merger Nash equilibrium is:

$$
\mathbf{p}=\left(\begin{array}{l}
265.163 \\
265.163 \\
5013.26
\end{array}\right), \mathbf{D}=\left(\begin{array}{c}
128.081 \\
128.081 \\
5013.26
\end{array}\right), \mathbf{\Pi}=\left(\begin{array}{c}
65,619.4 \\
25,132,757.2
\end{array}\right)
$$

After the merger, consumers' surplus is $S=-37,669,283.4$, while total welfare is $W_{1 / 2}=$ $-12,470,906.7$. The merger increases welfare by $3,269.4$. Moreover, it is privately profitable and, as expected, consumers' surplus is reduced.

\section{A.2 The profit of the merged entity can decrease with efficiency gains}

The intuition behind this result is fairly simple. Efficiency gains are not identical for all the varieties produced by the merged entity. Some marginal costs might (almost) not be affected by the merger while others might be significantly reduced. The prices of all products, however, decline after the merger as soon as some efficiency gains exist. That is, the profit margin of the merged entity might decline for some of its products and these profit losses might not be compensated by gains on other brands. To illustrate, take $n=3$ monoproduct firms and consider the following demand system:

$$
\left\{\begin{array}{l}
D^{1}=800-\frac{3}{5} p_{1}+\frac{1}{5,000} p_{2}+\frac{9}{20} p_{3} \\
D^{2}=10+\frac{1}{5,000} p_{1}-\frac{9}{20} p_{3} \\
D^{3}=10+\frac{9}{20} p_{1}+\frac{9}{20} p_{2}-p_{3}
\end{array}\right.
$$


Assume that the marginal costs of production are

$$
c_{1}=0, \text { and } c_{2}=c_{3}=5 .
$$

The pre-merger Nash equilibrium is:

$$
\mathbf{p}^{\text {pre }}=\left(\begin{array}{c}
735.70 \\
48.99 \\
184.0 .5
\end{array}\right), \mathbf{D}^{\text {pre }}=\left(\begin{array}{c}
441.42 \\
43.99 \\
179.05
\end{array}\right), \mathbf{\Pi}^{\text {pre }}=\left(\begin{array}{c}
324,748 \\
1,934.72 \\
32,060
\end{array}\right)
$$

Consumers' surplus is $S^{\text {pre }}=-475,372$; total welfare is $W_{1 / 2}^{\text {pre }}=-116,629$.

Suppose that firms 1 and 2 merge and generate efficiency gains: $c_{2}(\gamma)=5(1-\gamma)$. For $\gamma=0$, the post-merger Nash equilibrium is:

$$
\mathbf{p}=\left(\begin{array}{c}
735.71 \\
49.06 \\
184.07
\end{array}\right), \mathbf{D}=\left(\begin{array}{c}
441.42 \\
43.92 \\
179.07
\end{array}\right), \mathbf{\Pi}=\left(\begin{array}{c}
326,690 \\
32,067.5
\end{array}\right), S=-475,386
$$

and $W_{1 / 2}=-116,628$. Therefore total welfare decreased by 13.94 after the merger, which is privately profitable.

It is readily confirmed ${ }^{13}$ that, for $\gamma=\hat{\gamma}_{1} \approx 4.1 \%$, consumers' surplus is unaffected by the merger, $\Pi^{1+2}=326,690, \Pi^{3}=32,058$ and $W=-116,624>-116,629$. That is, the merger benefits society as a whole, consumers are indifferent (they might be made slightly better of), the merged entity profit increases and the outsider profit decreases. Yet, this merger is problematic because the profit of the merged entity $\Pi^{1+2} \approx 326,690.15-8.61 \gamma+$ $5.58 \gamma^{2}$ is a decreasing function of $\gamma$, for $\gamma \in[0,0.77]$. Once the merger is authorized, the merged entity would have no incentive to implement the efficiency gains (it seems reasonable to assume that while the merged entity cannot achieve gains greater than $\gamma$, it could nevertheless achieve gains lower than $\gamma$ ). But, absent efficiency gains, consumers' surplus and total welfare would fall (relatively to the pre-merger situation). If the merging firms cannot credibly commit to reduce their costs, antitrust authorities should block a would be profitable merger. All this has a prisoner dilemma flavor.

\section{A.3 Total welfare can decrease with efficiency gains}

Take $n=4$ monoproduct firms, and consider the following demand system:

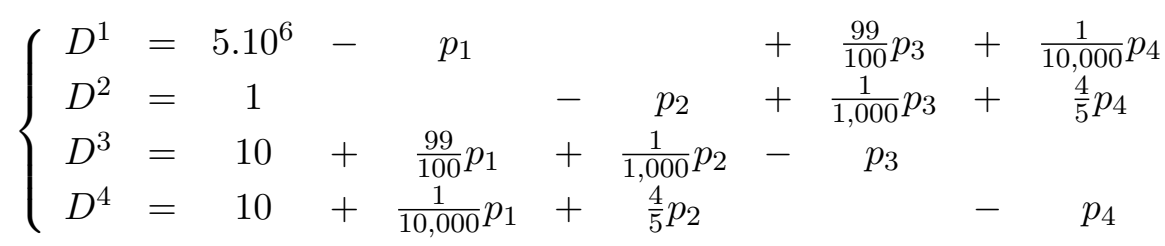

${ }^{13} S \approx-475,386.00+340.34 \gamma+2.88 \gamma^{2}$ 
Assume that the marginal costs of production are

$$
c_{1}=c_{3}=c_{4}=0 \text { and } c_{2}=1 / 2 \text {. }
$$

As $D^{1}$ (resp. $D^{2}$ ) is independent of $p_{2}$ (resp. $p_{1}$ ), for $\gamma=\hat{\gamma}_{1}=0$, the post-merger Nash equilibrium is identical to the pre-merger Nash equilibrium (no unilateral effects):

$$
\mathbf{p}^{\text {pre }}=\mathbf{p}=\left(\begin{array}{c}
3,311,371.59 \\
1,057.79 \\
1,639,134.46 \\
593.68
\end{array}\right), \mathbf{D}^{\text {pre }}=\mathbf{D}=\left(\begin{array}{c}
3,311,371.59 \\
1,057.29 \\
1,639,134.46 \\
593.68
\end{array}\right)
$$

Firms 1 and 2 merge and generate efficiency gains: $c_{2}(\gamma)=(1-\gamma) / 2$. The post-merger total welfare is given by

$$
W_{1 / 2}(\gamma) \approx-1.45 .10^{12}-6.72 \gamma+0.078 \gamma^{2}
$$

which is a non increasing function of $\gamma$ for $\gamma \in]-\infty, 1$ ] (if $\gamma$ is too negative, brand 2 is no longer produced). Thus, total welfare is decreasing in the magnitude of efficiency gains on the whole relevant range, that is, up to $100 \%$ gains. Note that in this example, the profit $\Pi^{1+2}$ of the merged entity is also a non increasing function of $\gamma$. The merger is, however, privately beneficial from an ex ante perspective if the firms' expectations put enough weight on $\gamma<0$.

\section{B Linear demand system}

In this section, we derive the best reply maps for linear demand systems and solve for the Nash equilibrium prices. Then, we examine the variations of prices and consumers' surplus with the magnitude of efficiency gains.

\section{B.1 Best reply map and Nash equilibrium prices}

Let $\boldsymbol{\Delta}$ denote the block diagonal matrix, whose blocks are $\mathbf{D}_{\mathbf{K}}^{\mathbf{K}}, K=I, J_{1}, \ldots, J_{r}$ and let $\mathbf{I}_{\mathbf{n}}$ denote the $n \times n$ identity matrix. The matrix $\boldsymbol{\Delta}$ has the same properties as the matrix B: it is symmetric negative definite.

For all $K, K=I, J_{1}, \ldots, J_{r}$, equation (4) rewrites $\mathbf{m}^{\mathbf{K}}=-\left(\mathbf{D}_{\mathbf{K}}^{\mathbf{K}}\right)^{-1} \mathbf{D}^{\mathbf{K}}$ that is:

$$
\mathbf{m}+\boldsymbol{\Delta}^{-\mathbf{1}} \mathbf{D}=0 \quad \text { or } \quad \mathbf{p}-\mathbf{c}+\boldsymbol{\Delta}^{-\mathbf{1}}(\mathbf{B} \mathbf{p}+\mathbf{A})=0 .
$$

It follows that the best reply map, which gives prices of each firm as functions of the prices of the other firms, writes

$$
\mathcal{R}(\mathbf{p})=\frac{1}{2} \mathbf{c}-\frac{1}{2} \boldsymbol{\Delta}^{-1} \mathbf{A}+\mathbf{R} \mathbf{p}
$$


where $\mathbf{R}$ is the constant $n \times n$ matrix given by

$$
\mathbf{R}=\frac{1}{2}\left(\mathbf{I}_{\mathbf{n}}-\boldsymbol{\Delta}^{-1} \mathbf{B}\right)
$$

Note that the diagonal blocks of the matrix $\mathbf{R}$ are zero, so that each price given by (16) only depends on the prices of goods controlled by other firms. ${ }^{14}$ Strategic complementarity is equivalent to each term of $\mathbf{R}$ being non negative.

The Nash equilibrium vector price is defined by: $\mathbf{p}=\mathcal{R}(\mathbf{p})$. So there exists a unique interior equilibrium if and only if the matrix $\mathbf{I}_{\mathbf{n}}-\mathbf{R}$ is nonsingular and the demand is positive for each good at the solution to $\mathbf{p}=\mathcal{R}(\mathbf{p})$. The Nash equilibrium vector price is given by

$$
\mathbf{p}=\left(\mathbf{I}_{\mathbf{n}}+\boldsymbol{\Delta}^{-1} \mathbf{B}\right)^{-1}\left(\mathbf{c}-\boldsymbol{\Delta}^{-1} \mathbf{A}\right)=\frac{1}{2}\left(\mathbf{I}_{\mathbf{n}}-\mathbf{R}\right)^{-1}\left(\mathbf{c}-\boldsymbol{\Delta}^{-1} \mathbf{A}\right) .
$$

Differentiating equation (17) yields:

$$
\dot{\mathbf{p}}=\frac{1}{2}\left(\mathbf{I}_{\mathbf{n}}-\mathbf{R}\right)^{-1} \dot{\mathbf{c}} .
$$

It follows that $\dot{\mathbf{p}} \neq 0$ unless if $\dot{\mathbf{c}}=0$.

\section{B.2 First derivative properties (proof of Lemma 1)}

In this subsection, we assume that the best reply map $\mathcal{R}($.$) is a contraction. This is$ equivalent to $\|\mathbf{R}\|<1$, where $\|\mathbf{R}\|$ is any matrix norm. ${ }^{15}$ Under this assumption, we can write

$$
\left(\mathbf{I}_{\mathbf{n}}-\mathbf{R}\right)^{-1}=\sum_{t=0}^{+\infty} \mathbf{R}^{t}
$$

Now, using equation (18) yields:

$$
\dot{\mathbf{p}}=\frac{1}{2}\left(\sum_{t=0}^{+\infty} \mathbf{R}^{t}\right) \dot{\mathbf{c}} \leq 0
$$

since all elements of $\mathbf{R}$ are nonnegative (strategic complementarity) and all components of $\dot{\mathbf{c}}$ are nonpositive. It follows that the consumers' surplus (the profit of each outsider $J$ respectively) is nondecreasing (resp. nonincreasing) in $\gamma$ :

$$
\dot{S}=-\mathbf{D} \cdot \dot{\mathbf{p}} \geq 0 \text { and } \dot{\Pi}^{J}=2\left(\mathbf{D}^{\mathbf{J}}\right)^{\prime}\left(\dot{\mathbf{p}}^{\mathbf{J}}\right)^{\prime} \leq 0,
$$

which completes the proof of Lemma 1. Note that the contraction assumption and the strategic complementarity are only sufficient assumptions for these results to hold.

\footnotetext{
${ }^{14}$ The off-diagonal blocks of $\mathbf{R}$ are $\mathbf{R}_{\mathbf{K}, \mathbf{H}}=\frac{1}{2}\left(-\mathbf{D}_{\mathbf{K}}^{\mathbf{K}}\right)^{-1} \mathbf{D}_{\mathbf{H}}^{\mathbf{K}}$ for $K \neq H, K, H=I, J_{1}, \ldots, J_{r}$.

${ }^{15} \mathrm{~A}$ matrix norm is defined as $\|\mathbf{R}\|=\sup _{\|\mathbf{x}\| \leq 1}\|\mathbf{R x}\|$, where $\|$. $\|$ stands for any norm of $\mathbb{R}^{n}$. For instance, consider the norm: $\|x\|_{\infty}=\max _{i}\left|x_{i}\right|$. The associated matrix norm is $\|\mathbf{R}\|_{\infty}=\max _{i} \sum_{j}\left|r_{i j}\right|$, where $r_{i j}$ is the generic element of $\mathbf{R}$. Using this norm, we checked that the best reply maps in all three examples of Appendix A are contractions.
} 


\section{B.3 Multidimensional gains}

In this subsection, we note $\frac{\mathrm{d}}{\mathrm{d} \boldsymbol{\gamma}}$ and $\frac{\mathrm{d}^{2}}{\mathrm{~d} \boldsymbol{\gamma}^{2}}$ the first and second derivatives of a scalar function with respect to the vector $\gamma$ of efficiency gains. For instance, $\frac{\mathrm{d} S}{\mathrm{~d} \gamma}$ is a column vector of size $l$ and $\frac{\mathrm{d}^{2} S}{\mathrm{~d} \boldsymbol{\gamma}^{2}}$ is a symmetric $l \times l$ matrix, where $l$ is the number of goods controlled by the new entity. Since prices are linear in $\gamma$, we have

$$
\frac{\mathrm{d} S}{\mathrm{~d} \boldsymbol{\gamma}}=-\frac{\mathrm{d} \mathbf{p}^{\prime}}{\mathrm{d} \boldsymbol{\gamma}} D \quad \text { and } \quad \frac{\mathrm{d}^{2} S}{\mathrm{~d} \boldsymbol{\gamma}^{2}}=-\frac{\mathrm{d} \mathbf{p}^{\prime}}{\mathrm{d} \boldsymbol{\gamma}} D \frac{\mathrm{d} \mathbf{p}}{\mathrm{d} \boldsymbol{\gamma}^{\prime}},
$$

where the generic term of the matrix $\frac{\mathrm{d} \mathbf{p}^{\prime}}{\mathrm{d} \boldsymbol{\gamma}}$ is $\frac{\mathrm{d} p_{j}}{\mathrm{~d} \gamma_{i}}, 1 \leq i \leq l, 1 \leq j \leq n$. (We use $\frac{\mathrm{d} \mathbf{p}}{\mathrm{d} \boldsymbol{\gamma}^{\prime}}$ for the transposed matrix of $\frac{\mathrm{d} \mathbf{p}^{\prime}}{\mathrm{d} \boldsymbol{\gamma}}$.) Since $D$ is symmetric negative definite, the $l \times l$ matrix $\frac{\mathrm{d}^{2} S}{\mathrm{~d} \boldsymbol{\gamma}^{2}}$ is symmetric positive semi-definite. Similarly, Equation (6) extends to multidimensional gains

$$
\frac{\mathrm{d}^{2} \Pi^{J}}{\mathrm{~d} \boldsymbol{\gamma}^{2}}=2 \frac{\mathrm{d}\left(\mathbf{m}^{\mathbf{J}}\right)^{\prime}}{\mathrm{d} \boldsymbol{\gamma}}\left(-\mathbf{D}_{\mathbf{J}}^{\mathbf{J}}\right) \frac{\mathrm{d} \mathbf{m}^{\mathbf{J}}}{\mathrm{d} \boldsymbol{\gamma}^{\prime}}
$$

As in the one-dimensional case, the convexity results follow from the concavity of demand.

\section{Merger to monopoly}

\section{C.1 The $\Psi$ function}

Let $P($.$) denote the inverse demand function. From P(\Phi(p))=p$, we deduce that

$$
\Phi^{\prime}(p)=\frac{1}{P^{\prime}(\Phi(p))} \text { and } \Phi^{\prime \prime}(p)=\frac{-\left(\Phi^{\prime}(p)\right)^{2} P^{\prime \prime}(\Phi(p))}{P^{\prime}(\Phi(p))} .
$$

It follows that

$$
\Psi(p)=\frac{E}{\varepsilon}=\frac{-\Phi(p) \Phi^{\prime \prime}(p)}{\left(\Phi^{\prime}(p)\right)^{2}}=\frac{\Phi(p) P^{\prime \prime}(\Phi(p))}{P^{\prime}(\Phi(p))}=\Theta(\Phi(p)),
$$

where $\Theta(Q)=Q P^{\prime \prime}(Q) / P^{\prime}(Q)$. In words, $\Psi(p)$ is the elasticity of the slope $P^{\prime}(Q)$ of the inverse demand function, evaluated at $Q=\Phi(p)$.

\section{C.2 First and second order conditions}

The first order condition of the monopolist's problem $\frac{\partial \Pi^{I}}{\partial p}=0$ writes:

$$
\Phi(p)+[p-(1-\gamma) c] \Phi^{\prime}(p)=0 \text { or } \frac{p-(1-\gamma) c}{p}=\frac{1}{\varepsilon}
$$

The second order condition $\frac{\partial^{2} \Pi^{I}}{\partial p^{2}}<0$ writes: 


$$
2 \Phi^{\prime}(p)+(p-(1-\gamma) c) \Phi^{\prime \prime}(p)<0 \text { or }\left(\frac{p-(1-\gamma) c}{p}\right) \frac{p \Phi^{\prime \prime}(p)}{\Phi^{\prime}(p)}>-2 .
$$

It follows that the second-order condition is satisfied at the solution to (20) if and only if

$$
E>-2 \varepsilon \text { or } \Psi>-2 .
$$

\section{C.3 Convexity of $S$ and $W_{\alpha}$}

The first derivatives of the consumers' surplus and the monopolist's profit are:

$$
\dot{S}(\gamma)=-n \dot{p} \Phi(p) \quad \text { and } \quad \dot{\Pi}^{I}(\gamma)=n c \Phi(p)
$$

The second derivatives are:

$$
\ddot{S}(\gamma)=-n \ddot{p} \Phi(p)-n \dot{p}^{2} \Phi^{\prime}(p) \quad \text { and } \quad \ddot{\Pi} I(\gamma)=n c \dot{p} \Phi^{\prime}(p) .
$$

From the first order condition (20), if follows that

$$
\dot{p}=\frac{-c}{2+E / \varepsilon}=\frac{-c}{2+\Psi}
$$

and therefore (using that $\mathrm{d} \Psi / \mathrm{d} \gamma=\dot{p} \Psi^{\prime}$ )

$$
\ddot{p}=\frac{(\dot{p})^{3}}{c} \Psi^{\prime} .
$$

Using (23), $\ddot{S}$ becomes:

$$
\ddot{S}=\underbrace{n \dot{p}^{2} \Phi}_{>0}[\underbrace{\frac{\Psi^{\prime}}{2+\Psi}}_{?}+\underbrace{\frac{\varepsilon}{p}}_{>0}]
$$

Now, using

$$
\ddot{\Pi}^{I}(\gamma)=n c \dot{p} \Phi^{\prime}(p)=n \dot{p}^{2} \Phi(2+\Psi) \frac{\varepsilon}{p}
$$

The second derivative of the welfare function is obtained by combining $\ddot{S}$ and $\ddot{\Pi}$.

\section{C.4 Proof of Corollary 3 and Lemma 5}

First we prove Lemma 5. Note that for a Logit demand, $\partial D^{i} / \partial p_{i}=-D^{i}\left(1-D^{i}\right) / \sigma$ which simplifies the f.o.c. Let $p^{\text {pre }}$ denote the pre-merger Nash equilibrium price, namely the solution to

$$
(p-c)(1-\Phi(p))=\sigma
$$


Since $(p-c)(1-\Phi(p))$ increases in $p$, we know that $p^{\text {pre }} / \sigma$ increases in $c / \sigma$ and $p_{0} / \sigma$. The post merger price $p(\gamma)$ is solution to

$$
[p-(1-\gamma) c](1-n \Phi(p))=\sigma
$$

Therefore, efficiency gains can offset the unilateral effect if and only if there exists $\gamma \leq 1$ such that

$$
\left(p^{\text {pre }}-(1-\gamma) c\right)\left(1-n \Phi\left(p^{\text {pre }}\right)\right)=\sigma,
$$

which is equivalent to (taking the value of the left hand side for $\gamma=1$ )

$$
p^{\text {pre }}\left(1-n \Phi\left(p^{\text {pre }}\right)\right) \geq \sigma,
$$

and, using the pre-merger f.o.c. $\left(p^{\text {pre }}-c\right)\left(1-\Phi\left(p^{\text {pre }}\right)\right)=\sigma$ to eliminate $\Phi$, it comes

$$
-\left(p^{\mathrm{pre}}\right)^{2}+(\sigma+c) p^{\mathrm{pre}}+\frac{\sigma c}{n-1} \geq 0
$$

Let $p^{+}(c)$ denote the largest solution of the equation $-p^{2}+(\sigma+c) p+\frac{\sigma c}{n-1}=0$ (the lowest solution is negative). The expression of $p^{+}(c)$ is given by (11). Condition (24) is equivalent to $p^{\text {pre }} \leq p^{+}$which (using the fact that $(p-c)(1-\Phi)$ is an increasing function of $p$ ) is equivalent to

$$
\left(p^{+}(c)-c\right)\left[1-\Phi\left(p^{+}(c)\right)\right] \geq \sigma
$$

which simplifies to prove (10) and completes the proof of Lemma 5. We turn now to Corollary 3.

By Lemma 4, the consumers' surplus $S=W_{1}$ is convex at $\hat{\gamma}_{1}$ if and only if $\Phi\left(p\left(\hat{\gamma}_{1}\right)\right) \leq$ $1 /(2 n)$. By definition of $\hat{\gamma}_{1}: p\left(\hat{\gamma}_{1}\right)=p^{\text {pre }}$. Now observe that the function $\varphi($.$) defined by$ $\varphi(c)=\Phi\left(p^{\mathrm{pre}}(c)\right)$ is decreasing. Therefore the condition $\Phi\left(p^{\mathrm{pre}}\right)<1 /(2 n)$ is equivalent to $c>\bar{c}=\varphi^{-1}(1 /(2 n))$. The equation $\varphi(c)=1 /(2 n)$ combined with $\left(p^{\text {pre }}-c\right)\left(1-\Phi\left(p^{\text {pre }}\right)\right)=$ $\sigma$ gives the value of $\bar{c}$ and proves (9).

\section{Bertrand competition with homogenous goods}

This section presents a proof of Proposition 3. Before the merger, the objective is $W_{\alpha}^{\text {pre }}=$ $\alpha S(c)$. After the merger, the objective is given by

$$
W_{\alpha}(\gamma)= \begin{cases}\alpha S(c)+(1-\alpha) \gamma c D(c) & \text { if } \gamma \geq 0 \\ \alpha S((1-\gamma) c)-(1-\alpha) \gamma c D((1-\gamma) c) & \text { if } \gamma \leq 0\end{cases}
$$

The convexity of the consumers' surplus and the identity $S^{\prime}(p)=-D(p)$ yield, for $\gamma \leq 0$

$$
S((1-\gamma) c)-S(c) \geq D(c) \gamma c
$$


Let $F$ be the cumulative distribution function of the random variable $\gamma$. Using successively (25), the assumption that $(1-\gamma) c \leq p^{m}(c)$ for $\gamma \leq 0$ (no drastic efficiency loss) and $\mathbb{E} \gamma=\int_{\gamma \geq 0} \gamma \mathrm{d} F(\gamma)+\int_{\gamma \leq 0} \gamma \mathrm{d} F(\gamma)=0$, we get

$$
\begin{aligned}
\mathbb{E}_{\gamma}\left(W_{\alpha}\right)-W_{\alpha}^{\mathrm{pre}=} & \int_{\gamma \geq 0}\left[W_{\alpha}(\gamma)-W_{\alpha}^{\mathrm{pre}}\right] \mathrm{d} F(\gamma)+\int_{\gamma \leq 0}\left[W_{\alpha}(\gamma)-W_{\alpha}^{\mathrm{pre}}\right] \mathrm{d} F(\gamma) \\
= & (1-\alpha) c D(c) \int_{\gamma \geq 0} \gamma \mathrm{d} F(\gamma)+ \\
& \int_{\gamma \leq 0}\{\alpha[S((1-\gamma) c)-S(c)]-(1-\alpha) \gamma c D((1-\gamma) c)\} \mathrm{d} F(\gamma) \\
\geq & (1-\alpha) c D(c) \int_{\gamma \geq 0} \gamma \mathrm{d} F(\gamma)+ \\
& \int_{\gamma \leq 0}\left\{\alpha \gamma c D(c)-(1-\alpha) \gamma c D\left(p^{m}(c)\right)\right\} \mathrm{d} F(\gamma) \\
= & c\left[(1-\alpha) D(c)-\alpha D(c)+(1-\alpha) D\left(p^{m}(c)\right)\right] \int_{\gamma \geq 0} \gamma \mathrm{d} F(\gamma)
\end{aligned}
$$

which is non negative for any $\alpha \geq \hat{\alpha}=\frac{D(c)+D\left(p^{m}(c)\right)}{2 D(c)+D\left(p^{m}(c)\right)}$.

\section{E Convexity of $S$ under Cournot competition}

Consumers' surplus is

$$
S(\gamma)=\int_{0}^{Q(\gamma)} P(u) \mathrm{d} u-P(Q(\gamma)) Q(\gamma)
$$

whence

$$
\dot{S}(\gamma)=-\dot{Q} Q P^{\prime}(Q)
$$

From

$$
(r+1) P(Q(\gamma))+Q(\gamma) P^{\prime}(Q(\gamma))=(1-\gamma) c^{I}+\sum_{j \in J} c_{j},
$$

it follows that (by differentiating with respect to $\gamma$ )

$$
-\dot{Q} P^{\prime}(Q)=\frac{c^{I}}{r+2+\Theta(Q)}
$$

therefore, $\dot{S}$ becomes

$$
\dot{S}=\frac{c^{I} Q}{r+2+\Theta(Q)}
$$


and

$$
\begin{aligned}
\ddot{S}(\gamma) & =\frac{c^{I} \dot{Q}}{r+2+\Theta(Q)}+\frac{-c^{I} Q \dot{Q} \Theta^{\prime}}{(r+2+\Theta(Q))^{2}} \\
& =\frac{c^{I} \dot{Q}}{r+2+\Theta(Q)}\left[1-\frac{Q \Theta^{\prime}}{r+2+\Theta(Q)}\right]
\end{aligned}
$$

